\title{
A Crystalline Quark-Hadron Mixed Phase in Neutron Stars ${ }^{\dagger} \ddagger$
}

Norman K. Glendenning

Nuclear Science Division

Lawrence Berkeley Laboratory

Berkeley, California 94720

August 31,1994

Invited Paper

Nordic Workshop on Relativistic Heavy Ion Reaction

Theory.

Bergen, Norway, June 11-15, 1993

and

Joint Session, Divisions of Astro and Nuclear Physics,

American Physical Society,

Washington, April 18-22, 1994

and

The Harmony of Physics (in Honor of S. Belyaev's 70'th

Birthday)

Philadelphia, May 9-11, 1994

and

NATO Workshop on Hot Hadronic Matter (in Honor of

Hagedorn's 75 'th Birthday)

Divonne-les Bains, June 27 - July 1, 1994

\footnotetext{
'To be published in Physics Reports.

\This work was supported by the Director, Office of Energy Research, Office of High Energy and Nuclear Physics, Division of Nuclear Physics, of the U.S. Department of Energy under Contract DE-AC03-76SF00098.
} 


\section{DISCLAIMER}

This report was prepared as an account of work sponsored by an agency of the United States Government. Neither the United States Government nor any agency thereof, nor any of their employees, make any warranty, express or implied, or assumes any legal liability or responsibility for the accuracy, completeness, or usefulness of any information, apparatus, product, or process disclosed, or represents that its use would not infringe privately owned rights. Reference herein to any specific commercial product, process, or service by trade name, trademark, manufacturer, or otherwise does not necessarily constitute or imply its endorsement, recommendation, or favoring by the United States Government or any agency thereof. The views and opinions of authors expressed herein do not necessarily state or reflect those of the United States Government or any agency thereof. 


\section{DISCLAIMER}

Portions of this document may be illegible in electronic image products. Images are produced from the best available original document. 


\title{
A Crystalline Quark-Hadron Mixed Phase in Neutron Stars
}

\author{
Norman K. Glendenning \\ Nuclear Science Division \\ Lawrence Berkeley Laboratory \\ Berkeley, California 94720
}

\begin{abstract}
The mixed phase of a substance undergoing a first order phase transition has entirely different behavior according as the substance has more than one conserved charge or only one, as in the text book examples. In the latter case the pressure and nature of the phases are constants throughout the coexistence phase. For systems with more than one conserved charge (or independent component) we prove two theorems: (1) The pressure and the nature of the phases in equilibrium change continuously as the proportion of the phases varies from one pure phase to the other. (2) If one of the conserved charges is the Coulomb force, an intermediate-range order will be created by the competition between Coulomb and surface interface energy. Their sum is minimized when the coexistence phase assumes a Coulomb lattice of one phase immersed in the other. The geometry will vary continuously as the proportion of phases. We illustrate the theorems for a simple description of the hadron to quark phase transition in neutron stars and find a crystalline phase many kilometers thick. However the theorems are general and pertain to chemical mixtures, nuclear systems, either static as in stars or dynamic as in collisions, and have possible application to phase transitions in the early universe.
\end{abstract}

\section{Outline}

We shall prove the theorems spoken of in the abstract in the context of the hadron to quark phase transition in neutron stars. This is a pedagogical choice, albeit one with far reaching implications for pulsar observables, that will serve to emphasize the physics of first order phase transitions in what we refer to as 'complex' substances, - those with more than one conserved charge. The theorems are general and rigorous $[1,2]$.

The only natural place in the universe where quark matter may exist is in neutron stars. Ever since the pioneering work of Baym and Chin $[3,4]$ in the mid-seventies it has been debated whether the cores of neutron stars are composed of quark matter. But curiously throughout the original paper and all succeeding ones the true nature of the phase transition was never put at question. It was always described in complete analogy with the text-book example of the water-vapor transition. This, as I will show, is a very special 
example of a first order phase transition, and not at all general.

In the traditional way of looking at it, the core of the star is purely a quark gas, and the exterior regions a neutron matter liquid, with the inevitable thin solid crust of rather ordinary metals at the surface.

What I will show is that taking full account of the physics of a first order phase transition in a 'complex' substance, such as neutron star matter which has more than one conserved charge (baryon and electric), and the physical constraint of charge neutrality of stars in its unrestricted sense, that is to say, as a global and not a local constraint, the situation is much different and much richer $[1,2,5,6]$. From a consideration of the bulk energy, there should exist in the star a broad region that is in the coexistence phase, and taking account of the forces at play, most especially the isospin restoring force in nuclear matter, the Coulomb force, and the surface interface energy, this region should be a Coulomb lattice of quark matter spheres of negative charge immersed in a background of positively charged hadronic matter with size and spacing of sites that minimize the Coulomb and surface interface energies. At greater depth within the star, the roles of quark and confined hadronic matter are interchanged. Between, the geometric structure will vary through other geometries, - rods and slabs. The thickness of the solid crystalline region of the mixed quark-hadron phase in compact stars of somewhat different mass should vary considerably, lending individuality to them. We estimate the thickness of the solid region to be a few kilometers, extending from the center to several kilometers from the edge of the lighter ones while in the heavier ones the central portion is expected to be pure quark matter with the solid mixed phase region outside it and somewhat thinner.

Many observable properties of compact stars will be effected by this thick solid dense interior region, including transport properties, both thermal and electrical, and pulsar glitches are likely to be influenced by if not actually centered in this solid. Indeed, the phenomenology of a compact star is likely to be so strongly coupled to this broad solid region, that I venture to suggest that in time the most convincing evidence of the existence of the quark-hadron phase transition will come from observed phenomena of compact stars and not from laboratory experiments.

\section{Degree(s) of Freedom}

All research up until 1990, and some later, had made one or other of two idealizations which had the side-effect of freezing out a degree of freedom. Nature on the other hand exploits every degree of freedom to find the lowest energy state. Therefore all work of the last quarter century on this phase transition in neutron stars described an excited, unrealizable state of the star. It is easy to state precisely what the degree of freedom is in the case of a neutron star, and as easy to see the generalization to arbitrary systems. Stars must 
be electrically neutral. This is because they are bound by the gravitational force, and any net charge would reduce the binding. A charged star would simply acquire from the interstellar medium such charge as needed to bring it to its ground state. However charge neutrality is a global property. It does not at all imply that the charge density is identically zero. There are many examples. The neutron itself has finite charge distributions; - they simply integrate to zero. There is another more relevant example. At the surface of a star the pressure is zero. Therefore the iron on the surface of a neutron star is much like the atomic iron found here on earth at one atmosphere of pressure. But a little way into the interior, the pressure rises, bringing the nuclei closer together. The atomic structure is destroyed. The atoms become fully ionized. Nevertheless the short-range nuclear force holds the nuclei together at sub-nuclear density, rather than allowing them to fill space uniformly at low density. So we have positively charged nuclei in an almost uniform background of electrons. The lowest energy state is a Coulomb crystal [7]. The matter is neutral, but not of vanishing charge density. The system finds it energetically favorable to maintain neutrality globally but not locally, in accord with the nature of the internal force(s). In earlier works, charge neutrality was imposed locally, either implicitly [3] (by assuming identically chargeless composition for the star) or explicitly [4] (by enforcing charge neutrality separately on each phase in equilibrium. This had the undesired feature of rendering the charge chemical potential discontinuous at the boundary between phases.)

The effect of freezing out the degree of freedom associated with a nonuniform distribution of charge in a two component system is equivalent to reducing it to a one component system, such as water. We recall that giving the system access to a degree of freedom that has been frozen out, is either neutral or lowers the energy of the system!

\section{First Order Phase transition in Simple Systems}

So as to better understand the more interesting nature of the mixed phase in a 'complex' system, we recall briefly the characteristic features in a 'simple' system (which has only one conserved charge, or one independent component, the terminology depending on whether one is dealing in chemistry or physics). The water-vapor transition is a familiar example of a first order phase transition in a simple system. The single independent component is $\mathrm{H}_{2} \mathrm{O}$. The remarkable and well known features of the mixed or coexistence phase are: (1) The pressure remains constant on an isotherm for all proportions of the two phases. (2) The nature of each phase in equilibrium is identical for all proportions.

We illustrate the well known pressure relation of a one-component substance in Fig. 1. The coexistence region is H,Q. All points between these two simply represent different volume proportions of the two phases in the states 
Figure 1: Behavior of the pressure vs. density in the vicinity of a first order phase transition in a simple substance having one chemical potential corresponding to conserved baryon number. Points labeled $\mathrm{H}$ and $\mathrm{Q}$ mark the end of phase 1 and beginning of phase 2 .

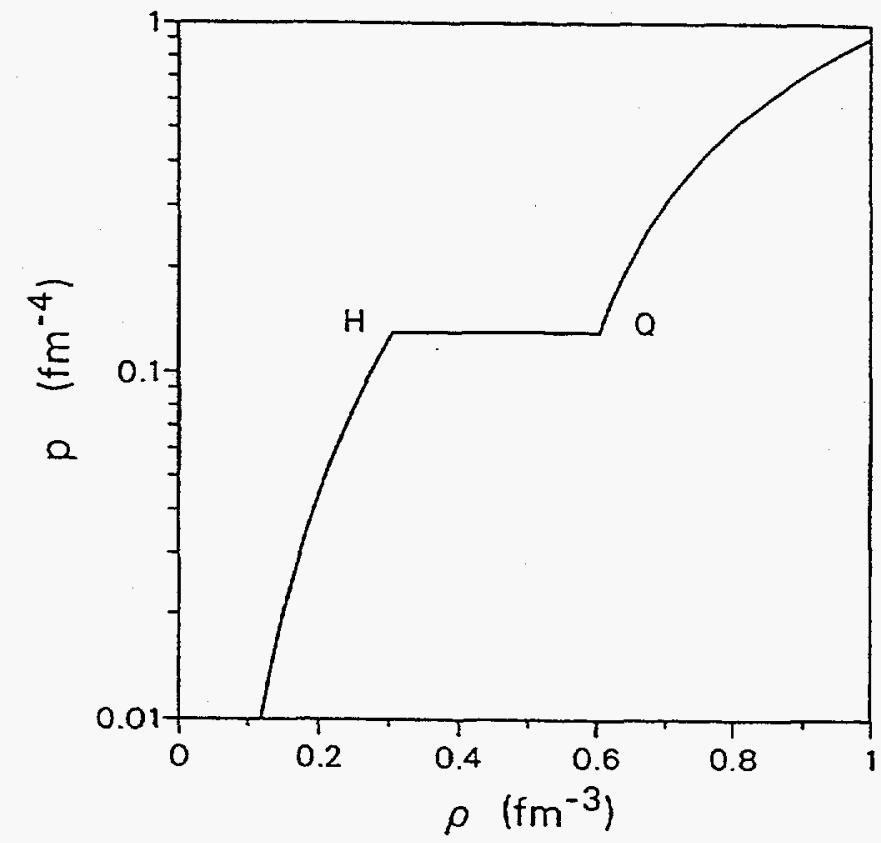

(their corresponding densities, and energies) denoted by $\mathrm{H}$ and $\mathrm{Q}$. The total energy is therefore a volume linear combination of two constants, and this is consistent with the constancy of the pressure since it is the negative of the volume derivative of the energy.

If this were the correct description of the hadron-quark phase transition in a neutron star it would have quite peculiar consequences. The equations of hydrostatic equilibrium, either classical or general relativistic, assure that the pressure is a monotonic decreasing function of distance from the center of the star, just as the pressure in our atmosphere is monotonic. Consequently the end points of the mixed phase, $\mathrm{H}$ and $\mathrm{Q}$ of Fig. 1, and all between, are mapped onto a single radial point in the star, as in Fig. 2. The densities of the two phases at $\mathrm{H}$ and $\mathrm{Q}$ are however different. So the mass-energy profile of a star, in a constant pressure transition is discontinuous as in Fig. 2.

This is completely analogous with water in equilibrium with its vapor in a heat bath in the presence of the earth's gravitational field, where we would plot $h$, the height above the bottom of the container instead of $r$. This is rigorously the only possibility for a simple system, one with one component! This analogy of the quark-hadron phase transition with the water-vapor transition has gone unchallenged until very recently $[1,2]$.

\section{First Order Transition in 'Complex' Systems}

There are profound differences between a first order phase transition with; (1) only one conserved charge. (simple system eg. $\mathrm{H}_{2} \mathrm{O}$ ).

(2) more than one. (complex system eg. ethane-heptane, neutron star). 


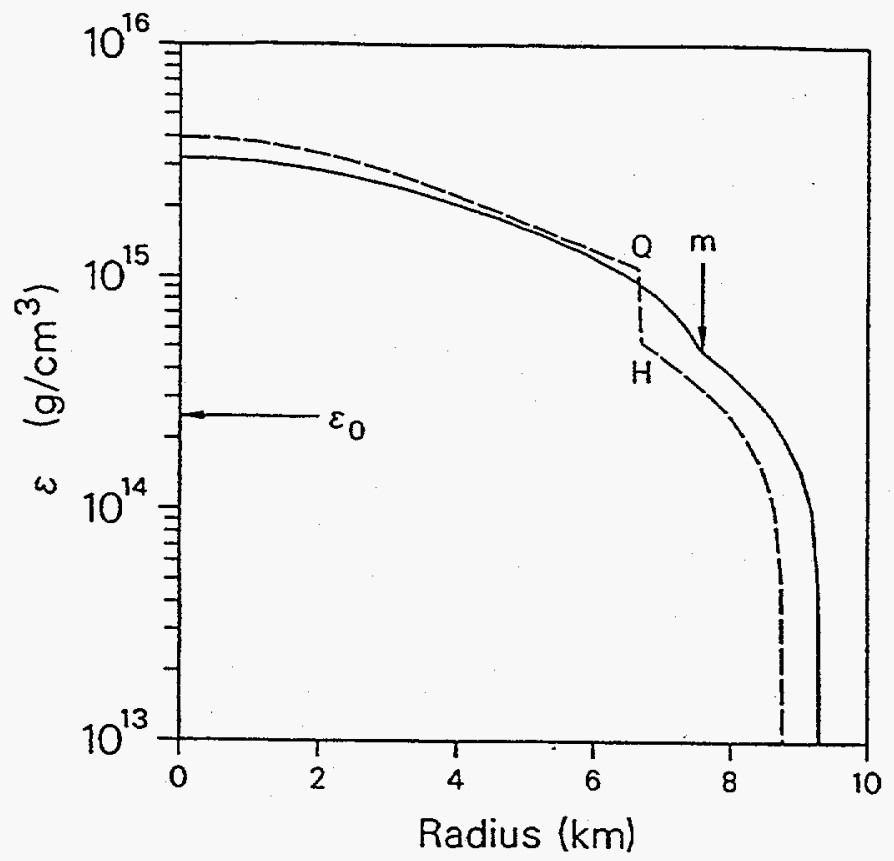

Figure 2: The mass-energy profile of a star showing the mapping of the points $H, Q$ of Fig. 1 in case the transition is treated as a simple one. The solid curve shows the profile when the transition is treated as the 'complex' one it is. The point ' $m$ ' marks the transition from mixed to pure hadronic phase.

(Conserved 'charge' means additive independent attribute like baryon number, electric charge, strangeness, number of $\mathrm{H}_{2} \mathrm{O}$, etc, etc.)

By one idealization or the other in earlier work, the transition in stars was inadvertently rendered as that of a simple system by one of the restrictions listed below, with the physically unacceptable consequence listed in parenthesis:

(1) Star is purely neutron, and converts to quark matter with the same types of quarks, $n_{d}=2 n_{u}$. (Both phases are $\beta$-unstable).

(2) Star is in $\beta$-equilibrium but is required to be everywhere locally charge neutral. (This leads to a discontinuous electron chemical potential at the interface of the phases and the constraint is inconsistent with Gibb's criteria!).

Both idealizations freeze out a degree of freedom available to a complex system which allows, even demands, that the pressure vary as the proportion of phases! I will explain this shortly. Moreover, both restrictions rigorously exclude the mixed phase from a star, as we have seen, whereas, with the restrictions lifted, the mixed phase is present and has a most intricate crystalline structure.

By the circumstances of its birth a neutron star has a fixed baryon charge, which being conserved, it keeps. Because of beta-equilibrium it also has charged particles even though the net charge is zero, as explained earlier. Therefore a neutron star has two conserved charges and two chemical potentials corresponding to them. We will now see what a difference this makes. 
Consider a substance composed of two conserved 'charges', - $Q$ of one kind, $B$ of the other. In the case of a star, these could denote the net electric charge number (in units of e) and baryon charge number. Let the substance be closed and in a heat bath. (This corresponds to the the loss of any energy produced in reaching the ground state of the star by neutrino and photon radiation.) Define their ratio (concentration),

$$
r=Q / B \text {. }
$$

Is this ratio fixed? One would certainly think so since $Q$ and $B$ are fixed. But the ratio is fixed only as long as the system remains in one pure phase or the other! However, when in the mixed phase the ratio in each of the regions of one phase or the other may be different and they are restricted only by the conservation on the total numbers,

$$
r^{\prime}=Q^{\prime} / B^{\prime}, \quad r^{\prime \prime}=Q^{\prime \prime} / B^{\prime \prime}, \quad\left(Q^{\prime}+Q^{\prime \prime}=Q, \quad Q^{\prime}+Q^{\prime \prime}=Q\right) .
$$

If the internal forces can lower the energy of the system by rearranging the ratio, it will be done.

In fact it is not possible to insist on a prescribed ratio and at the same time satisfy Gibbs' criteria. There are too many conditions for the number of variables [2].

The above observations allow us to prove easily the first theorem stated in the abstract. Consider the system at the density or pressure where the neutron star matter has just begun to condense some quark matter. There is little scope for the internal forces to optimize the concentrations, $r^{\prime}, r^{\prime \prime}$, in the two phases, since the small quantity of quark matter can neither receive nor donate much of either charge. However, at higher density or pressure, the proportion of the two phases will become more comparable, and the internal forces now have more scope to optimize the concentrations in the two phases, always consistent with overall conservation of the two charges. From this observation, we learn theorem (1) The nature of each phase in equilibrium changes with the proportion of the phases and since the total energy is now the volume proportion of the energy density of the two phases, each of which varies with the proportion, the derivative with respect to volume is no longer a constant. The pressure varies as the proportion of phases!

In contrast to a simple system, the mixed phase of a complex one is not squeezed out by gravity. It can occupy a finite radial extent in a star corresponding to the pressure spanned by the mixed phase!

\section{Role of an Internal Force}

We have spoken of the internal force(s) as driving the mixed phase to optimize the concentrations of the conserved charges in the two phases in equilibrium. We now discuss this explicitly, again in terms of a neutron star. 
In nuclear matter there is an isospin symmetry restoring force. It arises in part because of the Fermi energies, which if equal for a neutron-proton system minimizes the sum of eigen-energies, and in part because any 3-component of isospin couples to the rho meson, and costs the system the energy of the rho condensate (proportional to the square of the isospin 3-component of the mean rho field strength). From the valley of beta stability we know the preference for symmetry in $N$ (neutron) and $Z$ (proton) numbers. Suppose a system was prepared with $\mathrm{N}>>\mathrm{Z}$ or, as in a neutron star, is required to obey this condition so that the Coulomb force does not disrupt the star. Then the part of the star that is in the pure hadronic phase is sitting high above the minimum in energy as far as the nuclear force is concerned because of its high isospin. However at some depth within the star where quark matter first condenses, the isospin symmetric nuclear force will be able to rearrange charges to make the hadronic phase more symmetric to the extent permitted by the conditions of overall conservation of charges and the minimal energy. The hadronic matter will become electrically more positively charged, and the quark matter will become negatively charged by a rearrangement of the quark Fermi surfaces, so as to achieve overall electric charge neutrality. Electrons may also permeate the mixed phase region but since they do not contribute to the baryon charge that the star possess by the accident of its birth, and since they simply cost energy if the system can neutralize itself among the baryon carrying charged fermions, they will tend to be quenched.

We note here that the constraint of local charge neutrality, which goes beyond what is required for Coulomb stability of a star, freezes out this degree of freedom.

\section{Inevitability of a Coulomb Lattice in the Mixed Phase}

We saw above the role of the internal force(s) in rearranging charges between phases in the coexistence phase so as to minimize the energy consistent with overall conservation of the charges of the system. When one of these corresponds to the long-range Coulomb force, as it does in a neutron star, an intermediate-range ordering of regions of the two phases will minimize the energy as we show.

Charged regions cannot become too extensive because of the repulsive Coulomb self-energy. So they will tend to divide into small regions. The surface interface energy will resist this. Hence we have theorem (2) At any proportion of the phases there is an optimum size and shape of the regions that will arrange themselves into a lattice so as to minimize the surface and Coulomb energies.

We consider the geometrical arrangement of the two phases in equilibrium a little more formally. The surface energy per unit volume of a quark drop of radius $r$ in a nuclear background of radius $R$, chosen so that there is zero net 
charge in $R$ (Wigner-Seitz cell) is

$$
E_{S} / V=\left[4 \pi r^{2} \sigma\right] /\left[(4 \pi / 3) R^{3}\right]=(3 \sigma \chi) / r \equiv S(\chi) / r,
$$

where for droplets, $\chi=(r / R)^{3}$. is the volume proportion of the quark phase. Likewise, while more involved to prove [7], the Coulomb energy per unit volume has the form,

$$
E_{C} / V=C(\chi) r^{2}
$$

Their sum is a minimum when $E_{S}=2 E_{C}$. These equations lead at once to

$$
r^{3}=S(\chi) /(2 C(\chi)) \text {. }
$$

Thus at each proportion $\chi$ a definite size of quark drops immersed in the nuclear matter is specified and through $\chi\left(=(r / R)^{3}\right.$ for droplets) a definite separation between sites. We note that the long-range of the Coulomb force is screened by the formation of the lattice.

The functions $C$ and $S$ and the proportion $\chi$, expressed in terms of the geometry of the one phase immersed in the other, have quite definite forms for each geometry, for droplets, which merge to form rods, which merge to form slabs, in analogy with the sub-nuclear crystal structure of nuclei immersed in an electron gas, which is believed to form the crust of a neutron star [7].

\section{Local vs Global Charge Neutrality}

Since a star is bound by gravity and any net charge would reduce its binding, a star is charge neutral. This is a global condition, not a local one. But one might imagine that it can be satisfied by either imposing the conservation of charge in the mixed phase in the local sense by demanding that both phases in equilibrium be separately charge neutral,

$$
q_{H}\left(\mu_{b}, \mu_{e}\right)=0, \quad q_{Q}\left(\mu_{b}, \mu_{e}\right)=0,
$$

or in the global sense,

$$
(1-\chi) q_{H}\left(\mu_{b}, \mu_{e}\right)+\chi q_{Q}\left(\mu_{b}, \mu_{e}\right)=0 .
$$

Since each of these procedures satisfies the constraint of charge neutrality of the star, one may consider the energetics to decide between them. As already explicit in our whole discussion, the second way of calculationally enforcing the physical constraint of charge neutrality allows the internal forces, - the isospinsymmetric nuclear force in the case of a star, - to redistribute charge so as to lower the energy. A system on which stronger constraints are imposed lies higher in energy. So the second way of expressing charge neutrality, (7), is the physical one. One can go further than this. We show that it is not consistent 
with Gibbs' criteria for phase equilibrium to specify the concentrations $r^{\prime}, r^{\prime \prime}$ for the two phases in equilibrium. Thermal, chemical and mechanical equilibrium demand that,

$$
p_{H}\left(\mu_{b}, \mu_{e}, T\right)=p_{Q}\left(\mu_{b}, \mu_{e}, T\right) .
$$

Let us see the problem with attempting to specify the charge densities separately in each phase in equilibrium with the other. Baryon number conservation might be expressed in the proportional form (or any other individual form for each phase that conserves baryon number)

$$
\rho_{H}\left(\mu_{b}, \mu_{e}\right)=(1-\chi) B / V, \quad \rho_{Q}\left(\mu_{b}, \mu_{e}\right)=\chi B / V .
$$

But now one has five conditions to satisfy, $(6,8,9)$ with only three unknowns, $\mu_{b}, \mu_{e}, V$ to do it with. Here $\rho_{B}, \rho_{Q}$ are the baryon number densities in the two phases and $V$ is the local volume, (not to be confused with the volume of the star. Rather it is the volume surrounding a sample spacetime point in the star within which the equivalence principle assures that spacetime is flat to arbitrary accuracy. We may imagine a sequence of volumes along a radial direction. The volumes $V$ contain $B$ baryons and zero net charge, and are smaller toward the center of the star. In this way we see the correspondence with the conventional discussion of a sample substance contained in a laboratory vessel of volume $V$ with a plunger that allows one to vary the volume and hence the pressure, and a surrounding heat bath to keep the substance at constant temperature and such local volumes in the star. The pressure in the case of a star is chosen according to the depth and the volume by the requirement that it contain $B$ baryons.)

Alternately, baryon number in the mixed phase can be conserved overall,

$$
(1-\chi) \rho_{H}\left(\mu_{b}, \mu_{e}\right)+\chi \rho_{Q}\left(\mu_{b}, \mu_{e}\right)=B / V .
$$

Now at any specified proportion of phases, $\chi$ the alternative way of global conservation has the unknowns, $\mu_{b}, \mu_{e}, V$ in just that number of equations $(7,8,10)$. So Gibbs' conditions can only be satisfied if the concentration of conserved charges are not specified for both phases in equilibrium. The substance, with its internal forces will choose concentrations that are energetically most favorable at each proportion, $\chi$, of the phases since obviously the chemical potentials depend on the proportion. This shows that all properties including the common pressure (8) in the mixed phase varies as the proportion of the phases. This formally proves our verbal proof of theorem 1.

The variation of pressure, chemical potentials and energy density through the mixed phase is shown in a sample calculation, Fig. 3. The particle populations in the maximum mass star are shown in Fig. 4 as a function of radial coordinate. In this case the pure quark phase is not achieved in the star. 


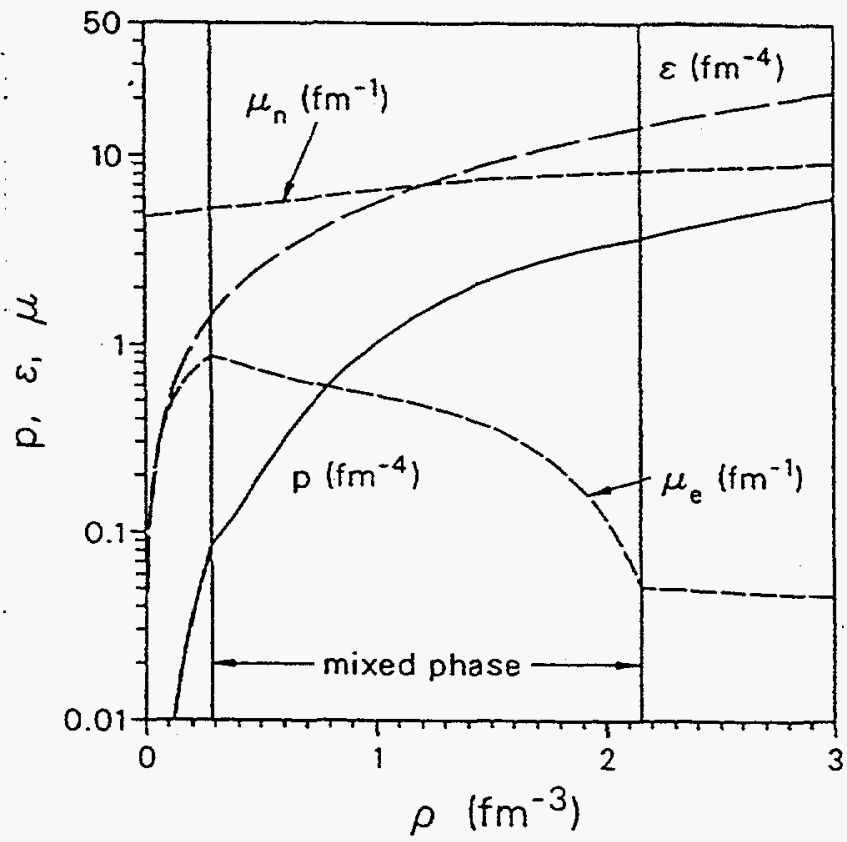

Figure 3: Showing the variation of the pressure in the mixed phase of a complex substance, in particular neutron star matter in the hadronic and quark matter phases. [2].

The central region of many kilometers of thickness is in the mixed phase and therefore is of varying crystalline structure.

\section{Calculational Expedient of Dividing Energy into Bulk and Surface}

Ideally one would like to compute the energy of a star allowing full freedom to find the lowest energy state of the Lagrangian or Hamiltonian used to describe its matter. Usually this is a very difficult problem especially when two phases may coexist and the internal force may rearrange the conserved charges optimally in the coexistence phase with a resultant formation of geometrical structure. The approximation of treating large systems in the bulk approximation, in which surface effects are at first ignored, and then later incorporated though a consideration of the surface and Coulomb energy of pieces of the bulk matter, perhaps in the Wigner-Seitz approximation, is well known and generally accurate. We are assured of this in the present case. The total energy is, $E_{\text {Total }}=E_{\text {Bulk }}+E_{\text {Surf. }}+E_{\text {Coul. }}$. The first term dominates and sets the radial scale of the mixed phase in the star. The Surface-Coulomb term alone is a fine variation about the first. The general equilibrium condition $E_{\text {Surf }}=2 E_{\text {Coul }}$ assures this. So for assessing the radial extension of the solid mixed phase in the star, the bulk properties dominate. The surface and Coulomb energies are important for studying the transport properties and strength of the solid.

As a matter of principle, we note that the less constrained way of imposing charge neutrality discussed in this paper, as a global condition and not as a local one must in general either be neutral or lead to a lower energy. Therefore 
Figure 4: Particle populations as a function of radial distance in a neutron star in ? which the mixed crystalline $E$ phase occupies most of the star from the center to about $7.5 \mathrm{~km}$. [2].

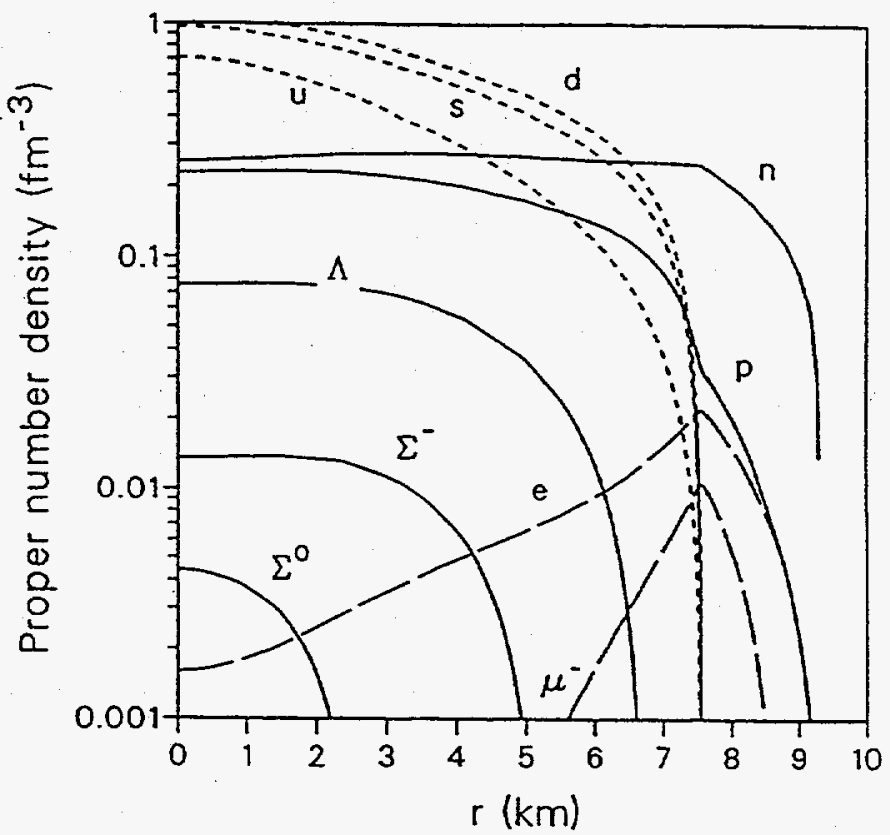

there is no question as to what range of surface energies will give the ordered phase a lower energy and what range a higher energy, a question raised in ref. [5]. The surface energy ought to be computed consistently with the bulk energies of the phases, and ought never to lead to a higher energy for the ordered crystal phase. In this sense we disagree with Heiselberg at al. who otherwise made a start on the study of the geometric structure [5] but use as arbitrary input, the surface interface energy coefficient, unrelated to the theories of the phases or the bulk energies of the phases and their difference.

We note that three authors using the presently described theory of a first order phase transition in a compact stars all agree that the mixed hadronicquark phase begins to appear at densities between 2-3 of normal nuclear matter density $[2,5,6]$. The original estimates of Baym and Chin placed the transition around 10 nuclear matter density. As explained in ref. [2], this was because highly excited states of both phases were used in the estimate.

\section{Outstanding Problems and Prospects}

It would appear that the presence of the coexistence phase in neutron stars leads to a host of interesting physical problems as well as observable consequences for pulsar observations, and should lead to highly individualistic behavior as regards glitches as an example, where the thickness of the interior solid region depends on the pressure profile and hence sensitively on the mass. Physical problems that need to be solved and associated pulsar observables are: 
(1) Surface interface energy between phases having a varying difference in energy density $(\sigma \neq$ const) (Needed for detailed calculation of lattice and lattice energy and relevant to pulsar glitches.)

(2) Detailed Coulomb lattice calculations (started by Heiselberg, Pethick and Staubo [5].

(3) Strength of the lattice, (sheer moduli), role of lattice defects. (Of great relevance to pulsar glitches, those irregularly-timed sudden changes in pulsar frequency.)

(4) Viscosity will be strongly modified, which impacts stability to fast rotation.

(5) Transport properties of a region of star interlaced by two media of different properties. (Electrical conductivity determines the decay constant of the magnetic field of pulsars and hence possibly controls their active lifetime.) (Cooling rate of neutron stars.)

This work was supported by the Director, Office of Energy Research, Office of High Energy and Nuclear Physics, Division of Nuclear Physics, of the U.S. Department of Energy under Contract DE-AC03-76SF00098.

\section{References}

[1] N. K. Glendenning, Nuclear Physics B (Proc. Suppl.) 24B (1991) 110.

[2] N. K. Glendenning, Phys. Rev. D, 46 (1992) 1274.

[3] G. Baym and S. A. Chin, Phys. Lett. 62B (1976) 241; B. D. Keister and L. S. Kisslinger, Phys. Lett. 64B (1976) 117; G. Chapline and M. Nauenberg, Phys. Rev. D 16(1977) 450; W. B. Fechner and P. C. Joss, Nature 274 (1978) 347; H. A. Bethe, G. E. Brown and J. Cooperstein, Nucl. Phys. A462 (1987) 791; B. D. Serot and H. Uechi, Ann. Phys. (N. Y.) 179 (1987) 272.

[4] N. K. Glendenning, in Proceedings of the 1989 International Nuclear Physics Conference, August 1989, Sao Paulo, Brasil, Vol. 2, p. 711, ed. by M. S. Hussein et al., (World Scientific, Singapore, 1990); N. K. Glendenning, in Relativistic Aspects of Nuclear Physics, August 1989, p. 241, ed. by T. Kodama et al., (World Scientific, Singapore, 1990); J. Ellis, J. Kapusta and K. A. Olive, Nucl. Phys. B348 (1991) 345; A. Rosenhauer, E. F. Staubo, L. P. Csernai, T. Overgard, and E. Ostgaard, Nucl. Phys. 540 (1992) 630.

[5] H. Heiselberg, C. J. Pethick, and E. F. Staubo, Phys. Rev. Lett. 70 (1993) 1355.

[6] V. R. Panharipande and E. F. Staubo, in International Conference on Astrophysics, Calcutta, 1993, ed. by B. Sinha, (World Scientific, Singapore).

[7] D. G. Ravenhall, C. J. Pethick and J. R. Wilson, Phys. Rev. Lett. 50 (1983) 2066; D. G. Ravenhall, C. J. Pethick and J. M. Lattimer, Nucl. Phys. A407 (1983) 571, and references cited. 